\title{
SDP-Based Robust Formation-Containment Coordination of Swarm Robotic Systems with Input Saturation
}

\author{
Kefan $\mathrm{Wu}^{1} \cdot$ Junyan $\mathrm{Hu}^{1}{ }^{1}$ - Barry Lennox ${ }^{1} \cdot$ Farshad Arvin $^{1}$ \\ Received: 24 August 2020 / Accepted: 12 March 2021 / Published online: 19 April 2021 \\ (C) The Author(s) 2021
}

\begin{abstract}
There are many potential applications of swarm robotic systems in real-world scenarios. In this paper, formation-containment controller design for single-integrator and double-integrator swarm robotic systems with input saturation is investigated. The swarm system contains two types of robots-leaders and followers. A novel control protocol and an implementation algorithm are proposed that enable the leaders to achieve the desired formation via semidefinite programming (SDP) techniques. The followers then converge into the convex hull formed by the leaders simultaneously. In contrast to conventional consensus-based formation control methods, the relative formation reference signal is not required in the realtime data transmission, which provides greater feasibility for implementation on hardware platforms. The effectiveness of the proposed formation-containment control algorithm is demonstrated with both numerical simulations and experiments using real robots that utilize the miniature mobile robot, Mona.
\end{abstract}

Keywords Swarm robotics $\cdot$ Formation-containment $\cdot$ Swarm controller design $\cdot$ Input saturation $\cdot$ Mobile robots

\section{Introduction}

In recent years, research studies on swarm robotics have generated increasing attention from the robotics and control communities. Swarm robotics mainly focuses on coordination control mechanisms within a group of homogeneous or heterogeneous robots by following collective and decentralized decision-making approaches that are mainly inspired by nature [1]. There are many swarm behaviors such as collective motion and flocking [2-5], aggregation [6, 7], foraging [8, 9], exploration [10],

\footnotetext{
Junyan $\mathrm{Hu}$

junyan.hu@manchester.ac.uk

Farshad Arvin

farshad.arvin@manchester.ac.uk

Kefan Wu

kefan.wu@manchester.ac.uk

Barry Lennox

barry.lennox@manchester.ac.uk
}

1 Swarm \& Computational Intelligence Lab (SwaCIL), Department of Electrical \& Electronic Engineering, The University of Manchester, Manchester, M13 9PL, UK and group transport [11], all of which have been successfully implemented using real mobile robots.

There are also many potential real-world applications for swarm robotic systems, such as object transportation [12, 13], autonomous shepherding [14], self-assembly [15], exploration of unknown environments [16, 17], agriculture [18, 19], and target surveillance [20, 21], all of which require carefully designed controllers, able to support limitations imposed by the physical environments (e.g., see Fig. 1).

A significant research direction in swarm robotics is formation control [22, 23], which explores how control systems can be designed to enable robots to converge to specific positions, allowing them to form the desired shape [24]. During the past decade or so, many control mechanisms have been proposed, and valuable results have been obtained that relate to formation control in swarm systems. As an example, [25] studied the consensus tracking problem for second-order swarm systems with switching interaction topologies, where a neighbor-based local controller and a neighbor-based state-estimation rule were proposed. In another study, [26] developed nonlinear gradient control laws for nonlinear multirobot systems. This work investigated the relationship between the cycle space of the formation graph and the resulting equilibria of cyclic formations. In [27], cooperative control of heterogeneous vehicle platoons using 
Fig. 1 A real-world scenario where leaders mark an area of interest using formation control. Followers then directly interact with plants, and follow the formation-containment algorithm

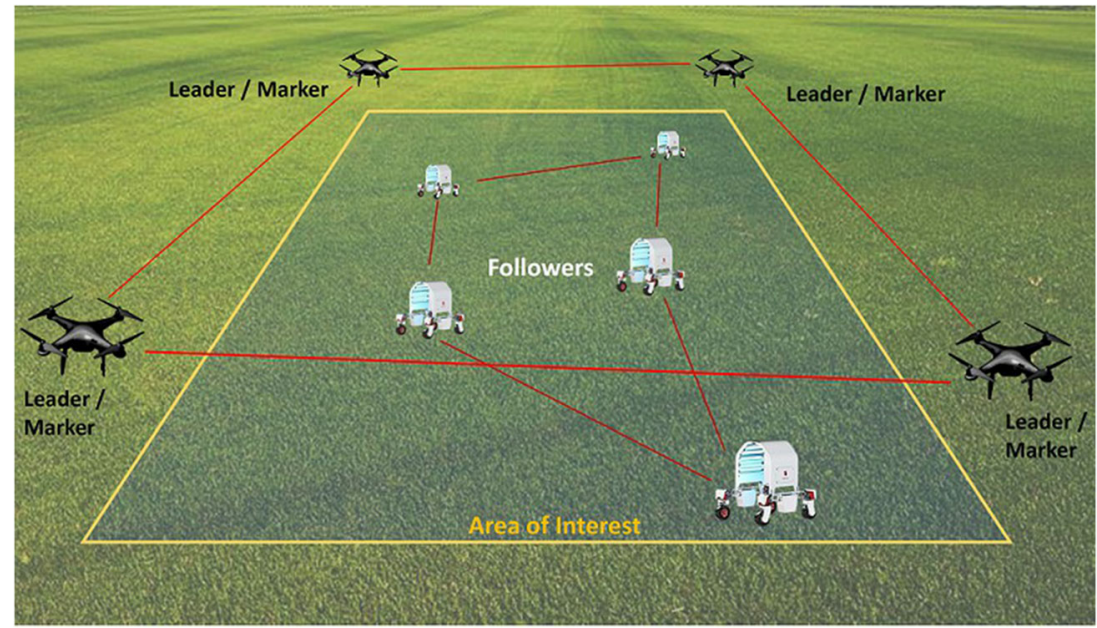

the adaptive formation control technique was investigated. Automatic cruise control was achieved via vehicle-tovehicle communication. Robust formation control of multiagent systems with uncertain dynamics was analyzed in [28], where the leader's nonzero control input was also considered in the controller design.

Most of the aforementioned literature only considered a single leader or leaderless scenarios. However, there are applications where two or more leaders, providing external control or group reference states for swarm systems, may exist and provide benefits. In the existence of multiple leaders, the containment problem should be analyzed where the followers can move into the convex hull formed by the states of the leaders. A hybrid stopgo control strategy was proposed in [29], which explored the concept of containment control. In another study, [30] considered distributed containment control for a doubleintegrator in both stationary and dynamic leaders, with two distributed containment control algorithms used in each scenario. In [31, 32], directed interaction topologies in containment problem were considered for high-order linear time-invariant (LTI) systems.

In the aforementioned research studies, it was assumed that there was no interaction between the leaders in the swarm. However, it is possible for the leaders to transmit information to other leaders to accomplish complex tasks, like formation control in real-world scenarios, such as agrirobotics. In these applications, it is desired that not only the followers can converge to the convex hull spanned by the leaders, but the leaders can also converge to the desired formation, which is formed as formationcontainment problems. In [33, 34], formation-containment problems for first-order swarm systems with undirected interaction topologies and switching interaction topologies were studied. Formation-containment problems for highorder LTI swarm systems were also discussed in [35].
In [36], adaptive formation-containment algorithms were proposed to coordinate multiple mobile robots. However, in the aforementioned works, the formation reference signal from the neighbors was required before designing the control protocol, which means that some global information is required. Hence, the formation-containment objectives cannot be achieved by only using range $\&$ bearing sensors in a real implementation, such as [37].

In this paper, we propose a novel distributed control protocol to solve the formation-containment problem in a collaborative swarm system. Both single-integrator dynamics and double-integrator dynamics are considered in the control protocol design. For each robot, the controller only requires the relative state information from its neighbors, which ensures the asymptotic stability of the swarm when controlling large scale robotic systems. The novelty of the control protocol for leaders is that it is robust in several conditions such as white noise perturbation, saturated input, etc, which has the potential to be used in extreme environments. The contributions of this paper are: (1) a novel formation-containment control framework which only requires local relative state measurements is proposed, (2) the convergence of the swarm system is guaranteed by the rigorous mathematical proof, and the feasibility of the proposed algorithm is analyzed, and (3) experiments using real robots were conducted to verify the effectiveness of the theoretical results.

The remainder of the paper proceeds as follows. Sections 2 and 3 present the proposed formation-containment framework, from which the swarm system under the proposed coordinated algorithm is shown to satisfy asymptotically stable properties via a rigorous mathematical proof. Section 4 presents simulation results and the experimental validation of the proposed strategy using laboratory-based small-scale mobile robots. Section 5 concludes the paper and discusses future research directions. 


\section{Preliminaries and Problem Description}

\subsection{Graph Theory}

Consider $N$ robots in $\mathbb{R}^{2}$. Let $x(t)$ be the position of robot $i \in\{1, \ldots, N\}$ at time $t$, and $x=\left[x_{1}^{\top}, \ldots, x_{N}^{\top}\right]^{\top}$ be the global state of all the robots. The interaction among the robots is described by a fixed graph $\mathscr{G}=(\mathscr{V}, \mathscr{E})$ which consists of a vertex set $\mathscr{V}=\left\{v_{1}, \ldots, v_{N}\right\}$ and an edge set $\mathscr{E} \subseteq\left\{\left(v_{i}, v_{j}\right): v_{i} ; v_{j} \in \mathscr{V}\right\}$. The edge of $\mathscr{G}$ is denoted by $e_{i j}=\left(v_{i}, v_{j}\right)$ and the weighted adjacency matrix with non-negative adjacency matrix elements $w_{i j}$ is denoted by $W=\left[w_{i j}\right] \in \mathbb{R}^{N \times N}$. The adjacency elements associated with the edge of $\mathscr{G}$ are positive, that is $e_{i j} \in \mathscr{E}$ if and only if $w_{j i}>0$. Besides, $w_{i i}=0$ for all $i \in\{1, \ldots, N\}$. The set of neighbors of nodes $v_{i}$ is represented by $\mathscr{N}=\left\{v_{j} \in\right.$ $\left.\mathscr{V}:\left(v_{j}, v_{i}\right) \in \mathscr{E}\right\}$. The degree matrix of $\mathscr{G}$ is defined as $D=\operatorname{diag}\left\{\operatorname{deg}_{i n}\left(v_{i}\right), i=1, \ldots, N\right\}$, where $\operatorname{deg}_{\text {in }}\left(v_{i}\right)=$ $\sum_{j=1}^{N} w_{i j}$ is the in-degree of node $v_{i}$. The Laplacian matrix of $\mathscr{G}$ is defined as $L=D-W$. A directed path from node $v_{i_{1}}$ to $v_{i_{l}}$ is a sequence of ordered edges with the form of $\left(v_{i_{k}}, v_{i_{k+1}}\right)$, where $l \in\{1, \ldots, N\}$ and $v_{i_{k}} \in \mathscr{V}(k=$ $1,2, \ldots, l-1)$. A directed graph is said to have spanning tree if there exists at least one node having a directed path to all the other nodes [38].

\subsection{Problem Description}

Robots in the swarm system can be divided into leaders and followers, with each having different properties: i) the neighbors of a leader are only leaders, and ii) the neighbors of a follower can be leaders or other followers. The leaders are expected to form the desired formation and the followers are expected to converge into the convex hull formed by the leaders by a proper design. Due to the behavioral differences, the swarm system can be viewed as a heterogeneous swarm as described by Dorigo et al. [19]. The communication network among all the robots is described using Graph Theory. We use $\mathscr{G}_{E}$ to represent the interaction topology among the leaders.

Assume that there are $M(M<N)$ followers and $N-M$ leaders in the robot swarm. Let $F=\{1,2, \ldots, M\}$ and $E=\{M+1, M+2, \ldots, N\}$ be the follower subscript set and leader subscript set respectively. Hence we have the follower's state $x_{i}(t)(\forall i \in F)$ and the leader's state $x_{j}(t)(\forall j \in E)$.

Definition 1 [35] A swarm system is said to achieve containment if for any given bounded initial states and any $k \in F$, there exists non-negative $\alpha_{k, j}(j \in E)$ satisfying $\sum_{j=M+1}^{N} \alpha_{k, j}=1$ such that

$\lim _{t \rightarrow \infty}\left(x_{k}(t)-\sum_{j=M+1}^{N} \alpha_{k, j} x_{j}(t)\right)=0$.

Let the formation reference vector $h \in \mathbb{R}^{2(N-M)}$ denote the coordinate of the desired formation of leaders and $h^{\perp} \in$ $\mathbb{R}^{2(N-M)}$ denote the coordinates of agents when the desired formation is rotated by $90^{\circ}$. The swarm system is said to achieve formation-containment if for any given bounded initial states, on condition that the agents of the leaders globally converge to the desired formation, there exists nonnegative $\alpha_{k, j}(j \in E)$ satisfying $\sum_{j=M+1}^{N} \alpha_{k, j}=1$ such that Eq. 1 holds.

\section{Formation-Containment Protocol Design}

\subsection{Formation-Containment Protocol Design for Single-Integrator Systems}

\subsubsection{Control Strategy}

In this section, we aim to design the formation-containment control protocol for single-integrator systems. Consider $N$ robots in the swarm system with single-integrator dynamics described by

$\dot{x}_{i}(t)=u_{i}(t)$,

where $x_{i}=\left[x_{i 1}, x_{i 2}\right]^{\top} \in \mathbb{R}^{2}$ is the coordinate for robot $i \in\{1,2, \ldots, N\}$, and $u_{i} \in \mathbb{R}^{2}$ is the control input. In order to achieve formation-containment, inspired by [39], the control protocol for the leaders and followers is chosen as

$u_{i}=\sum_{j \in \mathscr{N}_{i}} A_{i j}\left(x_{j}-x_{i}\right) \quad i \in E$,

$u_{i}=\sum_{j \in \mathscr{N}_{i}} w_{i j}\left(x_{j}-x_{i}\right) \quad i \in F$,

where $\mathscr{N}_{i}$ represents the neighbors of agent $i, w_{i j}$ is a nonnegative gain and $A_{i j} \in \mathbb{R}^{2 \times 2}$ are the constant control gain matrices with the form of

$A_{i j}=\left[\begin{array}{cc}a_{i j} & -b_{i j} \\ b_{i j} & a_{i j}\end{array}\right], \quad a_{i j}, b_{i j} \in \mathbb{R}$. 
In the formation control design process, we establish a formation matrix $A$ which contains the formation information and the communication topology of the leaders. $A$ is given by Eq. 6 as shown in the top of the next page,

$A=\left[\begin{array}{cccc}-\sum_{j=2}^{N-M} A_{1 j} & A_{12} & \ldots & A_{1(N-M)} \\ A_{21} & -\sum_{j=1, j \neq 2}^{N-M} A_{2 j} & \ldots & A_{2(N-M)} \\ \vdots & \vdots & \ddots & \vdots \\ A_{(N-M) 1} & A_{(N-M) 2} & \ldots & -\sum_{j=1}^{N-M-1} A_{(N-M) j}\end{array}\right] \in \mathbb{R}^{2(N-M) \times 2(N-M), \quad(6)}$

where $A_{i j}$ is defined as zero matrices if $j \notin \mathscr{N}_{i}$. We note that $A$ has block Laplacian structure, hence the following vectors

$\mathbf{1}=[1,0,1,0, \ldots, 1,0]^{\top} \in \mathbb{R}^{2(N-M)}$

$\mathbf{1}^{\perp}=[0,1,0,1, \ldots, 0,1]^{\top} \in \mathbb{R}^{2(N-M)}$

satisfy $A \mathbf{1}=0$ and $A \mathbf{1}^{\perp}=0$.

Denote $L$ as the Laplacian matrix corresponding to the interaction topology $(\mathscr{G})$ of the swarm system (2). $L$ has the form

$L=\left[\begin{array}{cc}L_{1} & L_{2} \\ 0 & L_{3}\end{array}\right]$,

where $L_{1} \in \mathbb{R}^{M \times M}, L_{2} \in \mathbb{R}^{M \times(N-M)}$ and $L_{3} \in$ $\mathbb{R}^{(N-M) \times(N-M)}$, the following Lemma holds.

Lemma 1 [40] Assume the interaction topology among leaders $\left(\mathscr{G}_{E}\right)$ has a spanning tree and, for each follower, there exists at least one directed path from a leader, we have:

(I) all eigenvalues of $L_{1}$ have positive real parts,

(II) each entry of $-L_{1} L_{2}$ is non-negative, and each row of $-L_{1} L_{2}$ has a sum of one.

Here, we present the following theorem to guarantee the convergence of a single-integrator system under the proposed formation-containment coordination.

Theorem 1 Consider leaders and followers with singleintegrator dynamics (2) and control protocol (3) and (4). Assume the interaction topology among leaders $\left(\mathscr{G}_{E}\right)$ contains a spanning tree and, for each follower, there exists at least one directed path from a leader, then, for any $w_{i j}>$ 0 , the swarm system (2) achieves formation-containment if $A_{i j}$ are selected such that

(I) $\mathbf{1}, \mathbf{1}^{\perp}, h$ and $h^{\perp}$ are linearly independent and $A \mathbf{1}=$ $A h=A \mathbf{1}^{\perp}=A h^{\perp}=0$,

(II) $\operatorname{Re} \lambda(A)<0$, where $\lambda(A)$ denotes the non-zero eigenvalues of $A$.

Proof Let $\bar{L}_{1}=L_{1} \otimes I_{2}$ and $\bar{L}_{2}=L_{2} \otimes I_{2}$, where $\otimes$ denotes the Kronecker product and $I_{2}$ denotes the identity matrix with two dimensions. By using control protocol (3) and (4), the system can be written as:

$\left(\begin{array}{c}\dot{x}_{F} \\ \dot{x}_{E}\end{array}\right)=\left[\begin{array}{cc}-\bar{L}_{1} & -\bar{L}_{2} \\ 0 & A\end{array}\right]\left(\begin{array}{l}x_{F} \\ x_{E}\end{array}\right)$,

where $x_{F}$ and $x_{E}$ are the states of followers and leaders, respectively.

On the one hand, for leaders, from (7), we have

$\dot{x}_{E}=A x_{E}$,

the solution of (8) can be shown as

$x_{E}(t)=e^{A t} x_{E}(0)$,

where $x_{E}(0)$ is the initial position of leaders.

Denote $J$ as the Jordan form of $A$, the following case is given on condition that $A$ has all Jordan blocks of order one. The general case follows similarly. Hence, we can obtain from (9) that

$$
\begin{aligned}
x_{E}(t) & =Q e^{J t} Q^{-1} x_{E}(0) \\
& =\sum_{i=1}^{2(N-M)} p_{i} e^{\lambda_{i} t} q_{i}^{\top} x_{E}(0) \\
& =\sum_{i=1}^{2(N-M)}\left(q_{i}^{\top} x_{E}(0)\right) e^{\lambda_{i} t} p_{i},
\end{aligned}
$$

where $p_{i} \in \mathbb{R}^{2(N-M) \times 1}$ and $q_{i}^{\top} \in \mathbb{R}^{1 \times 2(N-M)}$ are the right and left eigenvectors of $A$ respectively, and $\lambda_{i}(i=1,2, \cdots, 2(N-M))$ is the eigenvalues of $A$. From condition (I), we see that $1, \mathbf{1}^{\perp}, h$ and $h^{\perp}$ are four right eigenvectors respond to the four zero eigenvalues (denoted by $\lambda_{1}, \lambda_{2}, \lambda_{3}$ and $\lambda_{4}$, respectively). Furthermore, all the entries of the Jordan part of the four zero eigenvalues are zero. If we denote left eigenvectors of $\mathbf{1}, \mathbf{1}^{\perp}, h$ and $h^{\perp}$ are $q_{1}^{\top}, q_{2}^{\top} q_{3}^{\top}$ and $q_{4}^{\top}$, (10) can be written as

$$
\begin{aligned}
x_{E}(t)= & \sum_{i=5}^{2(N-M)}\left(q_{i}^{\top} x_{E}(0)\right) e^{\lambda_{i} t} p_{i}+\mathbf{1} q_{1}^{\top} x_{E}(0) \\
& +\mathbf{1}^{\perp} q_{2}^{\top} x_{E}(0)+h q_{3}^{\top} x_{E}(0)+h^{\perp} q_{4}^{\top} x_{E}(0) .
\end{aligned}
$$

From condition (II), we know

$\lim _{t \rightarrow \infty} e^{\lambda_{i} t}=0$ for any $i \geq 5$,

so we let $t \rightarrow \infty$ in (11), then we have

$$
\begin{aligned}
x_{E}(t) \rightarrow & \mathbf{1} q_{1}^{\top} x_{E}(0)+\mathbf{1}^{\perp} q_{2}^{\top} x_{E}(0) \\
& +h q_{3}{ }^{\top} x_{E}(0)+h^{\perp} q_{4}^{\top} x_{E}(0),
\end{aligned}
$$

if we denote $c_{1}=q_{1}^{\top} x_{E}(0), c_{2}=q_{2}^{\top} x_{E}(0), c_{3}=q_{3}^{\top} x_{E}(0)$ and $c_{4}=q_{4}^{\top} x_{E}(0)$, they are four constants, so we can get

$x_{E}(t) \rightarrow \mathbf{1} c_{1}+\mathbf{1}^{\perp} c_{2}+h c_{3}+h^{\perp} c_{4}$,

that is to say, the leaders can converge to nothing but all translations, rotations, and non-negative scale factors of $h$, 
which also indicates that leaders can converge to the desired formation shape.

On the other hand, for followers, from (7), we have

$\dot{x}_{F}=-\bar{L}_{1} x_{F}-\bar{L}_{2} x_{E}$.

Since leaders globally converge to the desired formation, denoted by $h_{F}$, let $t \rightarrow \infty$ in (14), we obtain

$\dot{x}_{F}=-\bar{L}_{1} x_{F}-\bar{L}_{2} h_{F}$,

then, we solve (15) and have

$$
\begin{aligned}
x_{F} & =e^{-t \bar{L}_{1}}\left(x_{F}(0)-\int_{0}^{t} e^{s \bar{L}_{1}} \bar{L}_{2} h_{F} \mathrm{~d} s\right) \\
& =e^{-t \bar{L}_{1}}\left(x_{F}(0)+\bar{L}_{1}{ }^{-1} \bar{L}_{2} h\right)-\bar{L}_{1}{ }^{-1} \bar{L}_{2} h_{F},
\end{aligned}
$$

where $x_{F}(0)$ is the initial position of the followers.

From Lemma 1 (I), all eigenvalues $L_{1}$ have positive real parts, that is to say, $L_{1}$ has no zero eigenvalue. Hence, $L_{1}$ is invertible and $\bar{L}_{1}$ is also invertible. We have

$x_{F}=-\bar{L}_{1}{ }^{-1} \bar{L}_{2} h_{F}$, when $t \rightarrow \infty$.

By Lemma 1 (ii), we can conclude that $x_{F}$ satisfies Definition 1, and then the swarm system (2) achieves formation-containment. This completes the proof.

Remark 1 It can be seen that for each robot, the formationcontainment protocol is fully distributed because the controller only requires the relative state information from its neighbors, which ensures the asymptotic stability of the swarm when controlling large-scale networked robots. One of the conditions of the proposed control strategy is the connectivity of the communication network. In the case when some robots are experiencing communication failures, if a proper network topology can be switched to connect all the robots, the robustness and stability of the whole swarm system can still be guaranteed as proved in Theorem 1. In the extreme situation where a robot loses communication with all the other robots, the remaining robots in the swarm will not be affected using the proposed coordination algorithm.

Remark 2 From Theorem 1, we also indicate that for any $w_{i j}>0$, the swarm system (2) achieves formationcontainment if and only if leaders globally converge to the desired formation.

Let $P=\left[h, h^{\perp}, \mathbf{1}, \mathbf{1}^{\perp}\right]$ and $U S V^{\top}=P$ be the singular value decomposition (SVD) of $P$, where

$U=[\bar{Q}, Q] \in \mathbb{R}^{2(N-M) \times 2(N-M)}$

with $\bar{Q} \in \mathbb{R}^{2(N-M) \times 4}$ defined as the first 4 columns of $U$ and $Q \in \mathbb{R}^{2(N-M) \times 2(N-M)-4}$ defined as the last $2(N-$ $M)-4$ columns of $U$.
Lemma 2 Using $Q$ in (18), define

$\bar{A}=\bar{Q} A Q \in \mathbb{R}^{(2(N-M)-4) \times(2(N-M)-4)}$.

Matrices $A$ and $\bar{A}$ have the same set of nonzero eigenvalues.

From Lemma 2, we can obtain that the projection operation in (19) removes the zero eigenvalues of $A$. By setting $a_{i j}=a_{j i}$ and $b_{i j}=-b_{j i}$ in (5) matrix $A$ can be design symmetric. Hence, its eigenvalues are real and can be ordered, then $A$ can be computed by solving the optimization problem

$A=\underset{a_{i j}, b_{i j}}{\operatorname{argmax}} \lambda_{1}(-\bar{A})$,

subject to $A P=0$,

where $\lambda_{1}(\cdot)$ denotes the smallest eigenvalue of a matrix.

From the Remark 2, in order to let the swarm system (2) achieve formation-containment, it is essential to design a proper $A$ matrix that satisfies the conditions in Theorem 1. Motivated by [41], we can use the following algorithm to solve this issue.

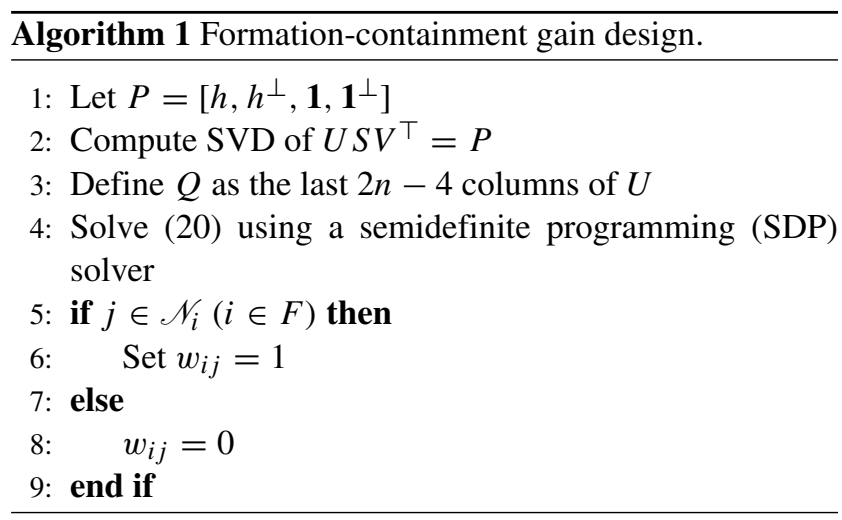

Remark 3 Even though the obstacle avoidance is not considered in the proposed formation-containment coordination protocol design, the standard low-level obstacle avoidance algorithms can be easily integrated with the proposed framework during the real-world operation. Based on different scenarios and tasks, the low-level controller which is implemented in the robot can be changed, however, the proposed formation-containment coordination algorithm will remain the same.

\subsubsection{Robustness to Input Saturation}

In real robotic systems, the velocity of each robot cannot exceed a certain value due to hardware constraints. Thus, any large control input will be saturated by a maximum allowed speed. This, however, does not affect the robots to accomplish the formation-containment task under the proposed control algorithm. 
In order to prove that the control protocols Eqs. 3 and 4 are robust to saturated inputs, the following Lemma is present here.

Lemma 3 [42] Consider the family of switched system $\dot{x}=f_{i}(x)$, with $i=1,2 \cdots, N$. Let $V: \mathbb{R}^{n} \rightarrow \mathbb{R}$ be a positive definite, continuously differentiable, and radially unbounded function. If $\frac{\partial V}{\partial x} f_{i}(x)<0, \forall x \neq 0, \forall i$ then the switched system is globally uniformly asymptotically stable.

Lemma 3 can be extended to the following corollary,

Corollary 1 For a positive semi-definite $V$ with the zero set of $Z:=\left\{x \in \mathbb{R}^{N}: V(x)=0\right\}$. In this case, If $\frac{\partial V}{\partial x} f_{i}(x)<0, \forall x \notin Z, \forall i$, then all trajectories globally uniformly asymptotically converge to $Z$.

Now, we present the theorem to show that the control protocols Eqs. 3 and 4 are robust to saturated input.

Theorem 2 Consider the single-integrator system (2), assume that $u_{\max }>0$ is a real positive scalar. If the control input $u_{i}$ of each robot is saturated such that $\left|u_{i}\right| \leq u_{\max }$, then under the control laws Eqs. 3 and 4, the formationcontainment can still be achieved globally.

Proof In order to model the saturated control input, we introduce the diagonal matrix $S \in \mathbb{R}^{2 N \times 2 N}$ with diagonal entries

$(S)_{i i}= \begin{cases}1 & \text { if }\left|u_{i}\right| \leq u_{\max } \\ \frac{u_{\max }}{\left|u_{i}\right|} & \text { if }\left|u_{i}\right|>u_{\max } .\end{cases}$

It can be seen that, the diagonal entries of $S$ are considered as functions that saturate any large control input to the maximum value $u_{\max }$. The single-integrator systems under saturated input can be expressed in the vector form via

$\left(\begin{array}{c}\dot{x}_{F} \\ \dot{x}_{E}\end{array}\right)=S\left[\begin{array}{cc}-\bar{L}_{1} & -\bar{L}_{2} \\ 0 & A\end{array}\right]\left(\begin{array}{c}x_{F} \\ x_{E}\end{array}\right)$.

From [42], Eq. 22 should be understood as a family of switched dynamical systems, and the solution is welldefined in the Filippov sense. To verify the stability of the system, two cases are discussed in the following part. Let

$S=\left[\begin{array}{cc}S_{F} & 0 \\ 0 & S_{E}\end{array}\right]$

where $S_{F} \in \mathbb{R}^{2 M \times 2 M}$ and $S_{E} \in \mathbb{R}^{2(N-M) \times 2(N-M)}$. Both $S_{F}$ and $S_{E}$ are diagonal matrices.

Part I (Leaders): The dynamics of the leaders can be described as

$\dot{x}_{E}=S_{E} A x_{E}$.
The Lyapunov function can be constructed by

$V_{E}=-\frac{1}{2} x_{E}^{\top} A x_{E} \geq 0$,

$V_{E}$ is a positive semi-definite scalar-valued function since $A$ is negative semi-definite. Time derivative of $V_{E}$ along the trajectory of Eq. 24 is

$$
\begin{aligned}
\dot{V}_{E} & =-x_{E}^{\top} A \dot{x}_{E} \\
& =-x_{E}^{\top} A S_{E} A x_{E} \\
& =-\left(S_{E}^{\frac{1}{2}} A x_{E}\right)^{\top}\left(S_{E}^{\frac{1}{2}} A x_{E}\right)=-\left\|S_{E}^{\frac{1}{2}} A x_{E}\right\|^{2} \leq 0,
\end{aligned}
$$

where $S_{E}^{\frac{1}{2}}$ is the diagonal matrix with entries given by the square root of diagonal elements of $S_{E}$. Note that all diagonal elements of $S_{E}$ are strictly positive, hence $S_{E}^{\frac{1}{2}}$ is well-defined. The last step of Eq. 26 is according to the fact that $A$ can be designed to be symmetric in Algorithm 1. Considering that $V_{E}$ is a positive semi-definite, continuously differentiable and radially unbounded function. Then, based on Lemma 3 , Corollary 1 and LaSalle's invariance principle, we can conclude that all trajectories of Eq. 24 converge to the zero set of $V_{E}$ (i.e., the kernel of $A$ ). That is to say, the formation of the leaders can be achieved under input constraints. The proof of the leaders' part is completed.

Part II (followers): Since the leaders can converge to the desired formation $h_{F}$, if $t$ is large enough, the dynamics of followers can be described as

$\dot{x}_{F}=-S_{F} \bar{L}_{1} x_{F}-S_{F} \bar{L}_{2} h_{F}$.

The Lyapunov function can be constructed by

$V_{F}=\frac{1}{2} x_{F}^{*} \bar{L}_{1} x_{F}^{*} \geq 0$,

where $x_{F}^{*}=x_{F}+\bar{L}_{1}{ }^{-1} \bar{L}_{2} h_{F} . V_{F}$ is a positive definite scalar-valued function since $\bar{L}_{1}$ is positive definite from Lemma 1 (i). Time derivative of $V_{F}$ along the trajectory of Eq. 27 is

$$
\begin{aligned}
\dot{V}_{F} & =x_{F}^{*} \bar{L}_{1} \dot{x}_{F} \\
& =\left(x_{F}+\bar{L}_{1}{ }^{-1} \bar{L}_{2} h_{F}\right)^{\top} \bar{L}_{1}\left(-S_{F} \bar{L}_{1} x_{F}-S_{F} \bar{L}_{2} h_{F}\right) \\
& =-x_{F}^{\top} \bar{L}_{1} S_{F} \bar{L}_{1} x_{F}-h_{F}^{\top} \bar{L}_{2}^{\top} S_{F} \bar{L}_{2} h_{F} \\
& =-\left(S_{F}^{\frac{1}{2}} \bar{L}_{1} x_{F}\right)^{\top}\left(S_{F}^{\frac{1}{2}} \bar{L}_{1} x_{F}\right)-\left(S_{F}^{\frac{1}{2}} \bar{L}_{2} h_{F}\right)^{\top}\left(S_{F}^{\frac{1}{2}} \bar{L}_{2} h_{F}\right) \\
& =-\left\|S_{F}^{\frac{1}{2}} \bar{L}_{1} x_{F}\right\|^{2}-\left\|S_{F}^{\frac{1}{2}} \bar{L}_{2} h_{F}\right\|^{2} \leq 0,
\end{aligned}
$$

where $S_{F}^{\frac{1}{2}}$ is the diagonal matrix with entries given by the square root of diagonal elements of $S_{F}$. Note that, all the diagonal elements of $S_{F}$ are strictly positive. Hence, $S_{F}^{\frac{1}{2}}$ is well-defined. The last step of Eq. 29 is according to the fact that $\bar{L}_{1}$ is symmetric for undirected interaction among followers. Similar to the above analysis, all 
trajectories of Eq. 27 converge to the zero set of $V_{F}$ applying Lemma 3, Corollary 1 and LaSalle's invariance principle. Hence, we can imply that

$$
x_{F}=-\bar{L}_{1}{ }^{-1} \bar{L}_{2} h_{F} \text {, when } t \rightarrow \infty \text {. }
$$

By Lemma 1 (ii), we can conclude that, $x_{F}$ satisfies Definition 1. Thus, the followers can converge to the hull convex formed by leaders. Combining Parts I and II, it can be concluded that the swarm system (2) achieves formation-containment under input saturation. This completes the proof.

\subsection{Formation-Containment Protocol Design for Double-Integrator Systems}

In this section, we extend the formation-containment protocol design with double-integrator systems in 2dimensions

$$
\begin{aligned}
& \dot{x}_{i}(t)=v_{i}(t) \\
& \dot{v}_{i}(t)=u_{i}(t),
\end{aligned}
$$

where $u_{i}(t)$ is the acceleration input to be designed. We can find that double-integrator systems (31) can be expressed in the following form

$$
\left(\begin{array}{c}
\dot{x}_{i} \\
\dot{v}_{i}
\end{array}\right)=\left[\begin{array}{cc}
0 & I_{2} \\
0 & 0
\end{array}\right]\left(\begin{array}{c}
x_{i} \\
v_{i}
\end{array}\right)+\left(\begin{array}{c}
0 \\
I_{2}
\end{array}\right) u_{i}
$$

Let $s_{i}=\left(x_{i}^{\top}, v_{i}^{\top}\right)^{\top}$, For the leaders and followers, our proposed formation-containment control protocol is

$u_{i}=\sum_{j \in \mathscr{N}_{i}} \hat{A}_{i j}\left(s_{j}-s_{i}\right) \quad i \in E$,

$u_{i}=\sum_{j \in \mathscr{N}_{i}} w_{i j}\left[\left(x_{j}-x_{i}\right)+\left(v_{j}-v_{i}\right)\right] \quad i \in F$,

where $\hat{A}_{i j}=\left[k_{0} A_{i j}, k_{1} A_{i j}\right], k_{0} \in \mathbb{R}$ and $k_{1} \in \mathbb{R}$ are scalar control gains. $w_{i j}$ are non-negative gain. The definition of $A_{i j}$ are shown in Eq. 5.

Now, we present the following theorem to guarantee the convergence of double-integrator systems under the proposed formation-containment coordination.

Theorem 3 Consider leaders and followers with doubleintegrator dynamics (31) and control protocol Eqs. 33 and 34. Assume the interaction topology among leaders $\left(\mathscr{G}_{E}\right)$ contains a spanning tree and, for each follower, there exists at least one directed path from a leader, then, for any $w_{i j}>$ 0 , the swarm system (31) achieves formation-containment if $A$ is that chosen by Algorithm 1, for all the non-zero eigenvalues of $A(\lambda(A)), k_{0}, k_{1}$ are selected such that

$\operatorname{Re}\left(k_{1} \lambda(A)+\Gamma\right)<0$,

where $\Gamma^{2}=k_{1}^{2} \lambda^{2}(A)+4 k_{0} \lambda(A)$.
Proof We put the control protocol Eqs. 33 and 34 into Eq. 32.

On the one hand, for leaders, we have

$\dot{S}_{E}=\left[\begin{array}{cc}0 & I_{2(N-M)} \\ k_{0} A & k_{1} A\end{array}\right] S_{E}$,

where, $S_{E}=\left[s_{M+1}^{\top}, \ldots, s_{N}^{\top}\right]^{\top}$. Let

$\hat{A}=\left[\begin{array}{cc}0 & I_{2(N-M)} \\ k_{0} A & k_{1} A\end{array}\right] \in \mathbb{R}^{4(N-M) \times 4(N-M)}$.

Then, the proof can be divided into two steps. In step 1, we will find the eigenvectors of $\hat{A}$ corresponding to zero. In step 2, we will show that all non-zero eigenvalues of $\hat{A}$ have negative real part.

Firstly, since

$\operatorname{Rank}(\hat{A})=\operatorname{rank}(A)+\operatorname{rank}\left(I_{2(N-M)}\right)=4(N-M)-4$,

we have the matrix $\hat{A}$ have 4 zero eigenvalues. If we let

$\hat{\mathbf{1}}=\left[\mathbf{1}^{\top}, 0\right]^{\top}, \hat{\mathbf{1}}^{\perp}=\left[\mathbf{1}^{\perp^{\top}}, 0\right]^{\top}$,

$\hat{h}=\left[h^{\top}, 0\right]^{\top}, \hat{h}^{\perp}=\left[h^{\perp^{\top}}, 0\right]^{\top}$,

we can easily verify that $\hat{A} \hat{\mathbf{1}}=\hat{A} \hat{\mathbf{1}}^{\perp}=\hat{A} \hat{h}=\hat{A} \hat{h}^{\perp}=0$. Hence we find the four eigenvectors corresponding to zero.

Secondly, since $A$ is selected by Algorithm 1, we have $\operatorname{Re}(\lambda(A))<0$, where $\operatorname{Re}(\lambda(A))$ denotes the non-negative eigenvalues of $A$. Then, the characteristic equation of $\hat{A}$ is given by

$$
\begin{aligned}
& \operatorname{det}\left(\lambda^{2} I_{2(N-M)}-k_{1} \lambda A-k_{0} A\right) \\
= & \prod_{\mu \in e i g(A)}\left|\lambda^{2}-k_{1} \mu \lambda-k_{0} \mu\right| \\
= & 0 .
\end{aligned}
$$

From Eqs. 35 and 37, for all non-zero eigenvalues of $\hat{A}$, we have

$\operatorname{Re}(\lambda(\hat{A}))=\frac{\operatorname{Re}\left(k_{1} \lambda(A)+\Gamma\right)}{2}<0$,

where $\Gamma^{2}=k_{1}^{2} \lambda^{2}(A)+4 k_{0} \lambda(A)$.

Therefore, we can conclude that all of the noneigenvalues of $\hat{A}$ have negative real part. Hence, similar to the discussion in the leader case of Theorem 1, when $t \rightarrow \infty$, we obtain

$S_{E}(t) \rightarrow \hat{\mathbf{1}} \hat{c}_{1}+\hat{\mathbf{1}}^{\perp} \hat{c}_{2}+\hat{h} \hat{c}_{3}+\hat{h}^{\perp} \hat{c}_{4}$,

which implies

$x_{E}(t) \rightarrow \mathbf{1} \hat{c}_{1}+\mathbf{1}^{\perp} \hat{c}_{2}+h \hat{c}_{3}+h^{\perp} \hat{c}_{4}$,

where $\hat{c}_{1}, \hat{c}_{2}, \hat{c}_{3}$ and $\hat{c}_{4}$ are constants. That is to say, the leaders can converge to nothing but all translations, rotations, and non-negative scale factors of $h$, which also indicates that leaders can converge to the desired formation shape. 
On the other hand, for followers, we have

$\dot{S}_{F}=\left[\begin{array}{cc}0 & I_{2 M} \\ -\bar{L}_{1} & -\bar{L}_{1}\end{array}\right] S_{F}+\left[\begin{array}{cc}0 & 0 \\ -\bar{L}_{2} & -\bar{L}_{2}\end{array}\right] S_{E}$,

where $S_{F}=\left[s_{1}^{\top}, \ldots, s_{M}^{\top}\right]^{\top}$. Let $t \rightarrow \infty$, similar to discussion in the follower case of Theorem 1, we conclude

$S_{F} \rightarrow\left[\begin{array}{cc}-\bar{L}_{1}{ }^{-1} \bar{L}_{2}-\bar{L}_{1}{ }^{-1} \bar{L}_{2} \\ 0 & 0\end{array}\right] \hat{h}_{F}$,

where $\hat{h}_{F}=\left[h_{F}^{\top}, 0\right]$ and $h_{F}$ is the formation shaped by leaders. Then we can imply

$x_{F}=-\bar{L}_{1}^{-1} \bar{L}_{2} h_{F}$, when $t \rightarrow \infty$.

From Lemma 1 (ii), we can conclude that $x_{F}$ satisfy Definition 1, and then the swarm system (31) achieve formation-containment. This completes the proof.

Since the condition (35) is not easy to be verified, we have the following corollary

Corollary 2 In Theorem 3, if we can select the matrix $A$ which satisfies $\lambda(A) \in \mathbb{R}$, where $\lambda(A)$ denote all non-zero eigenvalues of $A$. The chosen of $k_{0}$ and $k_{1}$ can be substituted by $k_{0}>0$ and $k_{1}>0$.

Proof Since $\lambda(A) \in \mathbb{R}$, we have $\Gamma^{2} \in \mathbb{R}$.

On the one hand, in Eq. 37, if $k_{1}^{2} \lambda^{2}(A)+4 k_{0} \lambda(A) \leq 0$, we can obtain

$\operatorname{Re}(\lambda(\hat{A}))=\frac{k_{1} \lambda(A)}{2}<0$.

On the other hand, in Eq. 37, if $k_{1}^{2} \lambda^{2}(A)+4 k_{0} \lambda(A)>0$,

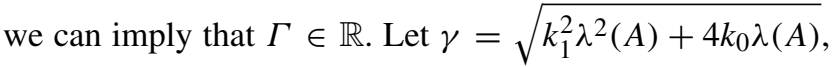
we have

$$
\begin{aligned}
\operatorname{Re}(\lambda(\hat{A})) & =\frac{k_{1} \lambda(A)+\Gamma}{2} \\
& <\frac{k_{1} \lambda(A)+\gamma}{2} \\
& =\frac{4 k_{0} \lambda(A)}{\gamma-k_{1} \lambda(A)} .
\end{aligned}
$$

Using this, combined with the selection of $A, k_{0}$ and $k_{1}$, we conclude that $\operatorname{Re}(\lambda(\hat{A}))<0$. The remaining proof is similar with Theorem 3, we omit here.

Remark 4 It can be seen that the formation matrix $A$ in the control protocol plays a key role in both single and double integrator systems. Different from [21, 31, 43], where both the relative formation reference signal and the relative state information are required in the real-time data transmission, the proposed control law only uses the relative state information. Hence, less data is used in the transmission results in reducing the communication cost in each robot.
Remark 5 The values of $k_{0}$ and $k_{1}$ reflect the convergence speed of velocity and acceleration, hence, if we select a larger set of $k_{0}$ and $k_{1}$, the formation-containment task will be completed faster. However, considering the energy costs and constraints of real-robot hardware, we cannot select arbitrary large values of $k_{0}$ and $k_{1}$. Therefore, there is a trade-off between control performance and input constraints.

\section{Results \& Discussion}

This section presents the observed results from simulation studies, followed by the results obtained from experiments using real-robots.

\subsection{Mission Description}

Formation-containment control of a swarm robotic system has the potential to be applied in various applications such as automated farming and precision agriculture [19, 44]. As an example, Fig. 1 shows an agri-robotic scenario using two different types of robots in a swarm, which are deployed for a weed management task. The leader robots (which are quadrotors with their embedded sensory system, e.g. multispectral imaging camera) are deployed to form a formation around the boundary of the target area i.e. an area of interest. The follower robots (which are the ground robots directly interacting with plants) converge to the target area spanned by the leaders using interrobot communication to complete the task, which may, for example, be the targeted application of insecticide. By developing and coordinating multiple sensing mobile platforms, the observed data can facilitate the practices of sustainable agricultural intensification.

\subsection{Simulation Results}

In this section, we design a numerical simulation in the Matlab/Simulink environment to demonstrate the effectiveness of the theorem results obtained in the previous section.

We consider the double-integrator swarm system with four leaders and ten followers. Their dynamics are described by Eq. 31. The interaction topology among them is shown in Fig. 2. To test the robustness of the system, we add the white noise with amplitude equals to 0.03 to the measured position of each robot. The input saturation is also considered in the simulation, where we set $\left\|u_{\max }\right\|=3$ for all the robots.

Initially, the desired formation of the four leader agents is chosen as a square. For simplicity, we set the final configuration of four agents as $(0,0),(1,0),(1,1)$ and 
Fig. 2 Interaction topology of the proposed swarm system. The four UAVs denote the leaders and the ten Mona robots denote the followers. The interactions among leaders and among followers are denoted by the red lines and yellow lines, respectively. The interactions between leaders and followers are denoted by blue arrows

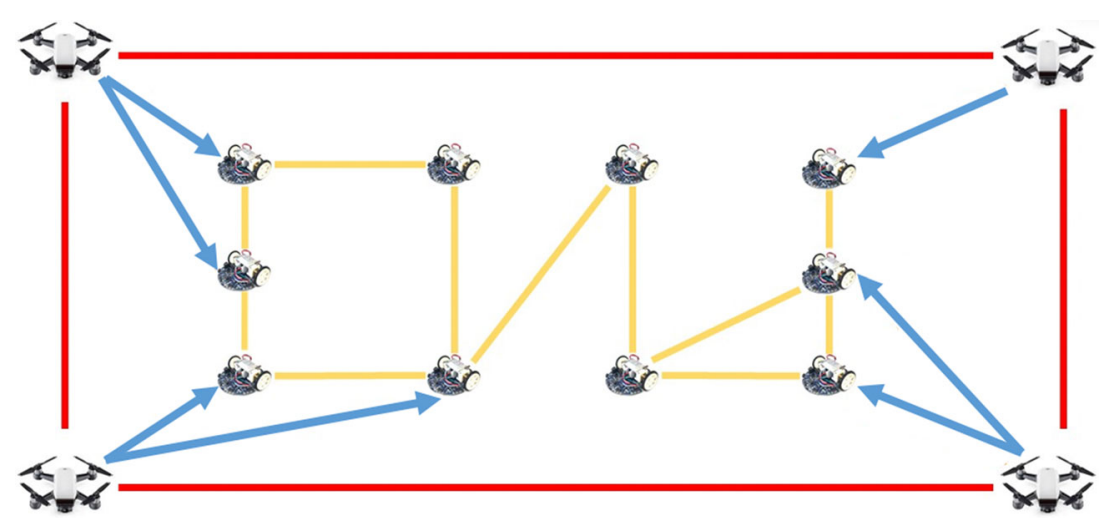

$(0,1)$ to fix a square shape. Hence, $h$ can then be defined as the following form $h=[0,0,1,0,1,1,0,1]^{\top}$.

Then, the matrix $A$ is generated as follows, utilising Algorithm 1

$$
A=\left[\begin{array}{cccccccc}
-2 & 0 & 1 & 1 & 0 & 0 & 1 & -1 \\
0 & -2 & -1 & 1 & 0 & 0 & 1 & 1 \\
1 & -1 & -2 & 0 & 1 & 1 & 0 & 0 \\
1 & 1 & 0 & -2 & -1 & 1 & 0 & 0 \\
0 & 0 & 1 & -1 & -2 & 0 & 1 & 1 \\
0 & 0 & 1 & 1 & 0 & -2 & -1 & 1 \\
1 & 1 & 0 & 0 & 1 & -1 & -2 & 0 \\
-1 & 1 & 0 & 0 & 1 & 1 & 0 & -2
\end{array}\right] .
$$

Finally, for followers, we set the interaction that has $0-1$ weight in the final step of Algorithm 1. It is straightforward to verify that all nonzero eigenvalues of $A$ are real. Hence, by Corollary 2, we set the control gain for $k_{0}=k_{1}=$ $\frac{1}{10}$. The swarm system (31) will achieve the formationcontainment by the control protocol Eqs. 33 and 34 .

Figure 3 shows the trajectory snapshots of leaders and followers with the interaction topology in Fig. 2 at different time instants $t \in\{0,20,50,100\}$ s. The positions of followers are denoted by circles, and the positions of leaders are denoted by square, triangle, diamond, and asterisk, respectively. Moreover, the convex hull formed by leaders is marked by the red solid line. Figure 3a shows the initial state of leaders and followers. From (a) to (b) in Fig. 3, it can be seen that the positions of the followers converged to the convex hull which was formed by the leaders. Figure $3 b-$ $\mathrm{d}$ show the leaders converged to the desired formation and, simultaneously, the followers still stayed in the convex hull formed by the leaders. Finally, the swarm system achieved formation-containment at $t=100 \mathrm{~s}$. The states and control inputs of the robots are shown in Fig. 4, where the solid lines denote leaders, and the dash lines denote followers. It can be seen that the velocity and acceleration of each robot converge to zero; and all the robots complete the formationcontainment task within $t=80 \mathrm{~s}$. Furthermore, the results of simulation reveal that our control protocol is robust in several situations like white noise perturbation and input constraints.

Next, we analyze how different cases in followers and leaders affected the result of the formation-containment. Two metrics which are i) time to achieve formationcontainment (denoted by $T$ ) and ii) the average distance between followers to the barycentre in followers (denoted by $d$ ) were investigated. We assumed there are four leaders in the swarm system with the interaction shown in Fig. 2, and the interaction topology between followers is a ring. The variables are the number of followers and the

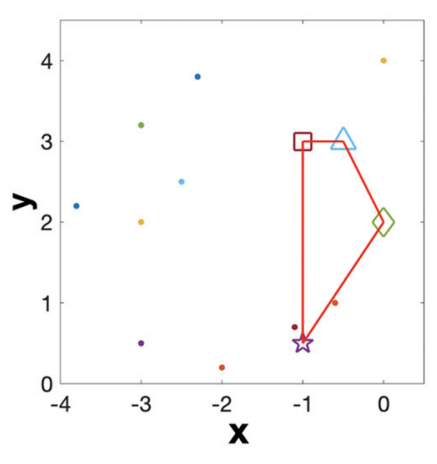

a

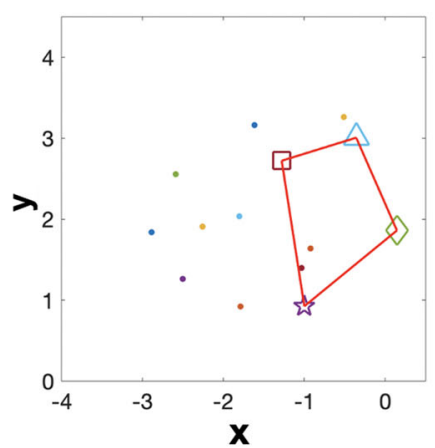

b

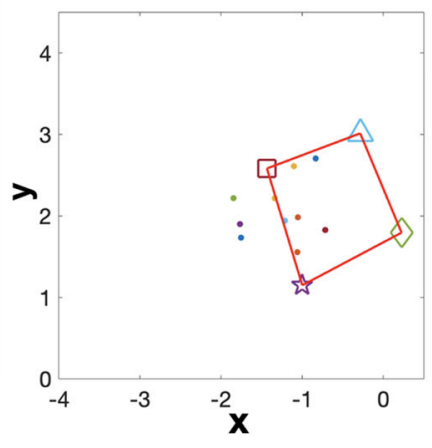

C

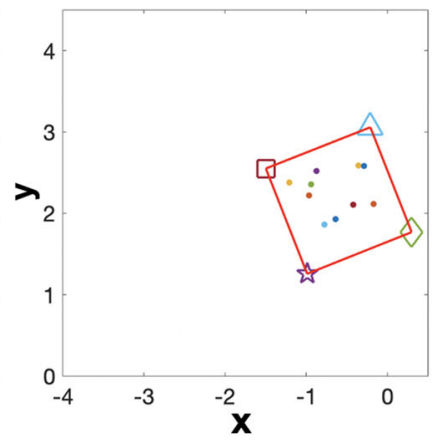

d

Fig. 3 Trajectory snapshots of leaders (denoted by square, triangle, diamond, and asterisk, respectively) and followers (denoted by ten nodes) in the swarm system at different time instants, $t \in\{0,20,50,100\} \mathrm{s}$ 
Fig. 4 a and $\mathbf{b}$ show the time variation of position in $\mathrm{x}$-axis $\left(p_{x_{i}}\right)$ and y-axis $\left(p_{y_{i}}\right)$ of $i$ th robot, respectively. $\mathbf{c}$ and $\mathbf{d}$ show the time variation of velocity in $\mathrm{x}$-axis $\left(v_{x_{i}}\right)$ and $\mathrm{y}$-axis $\left(v_{y_{i}}\right)$ of $i$ th robot, respectively. $\mathbf{e}$ and $\mathbf{f}$ show the time variation of acceleration (control input) in $\mathrm{x}$-axis $\left(a_{x_{i}}\right)$ and $\mathrm{y}$-axis $\left(a_{y_{i}}\right)$ with saturated input equals to 3 of $i$ th robot, respectively. The solid lines denote leaders, and the dash lines denote followers

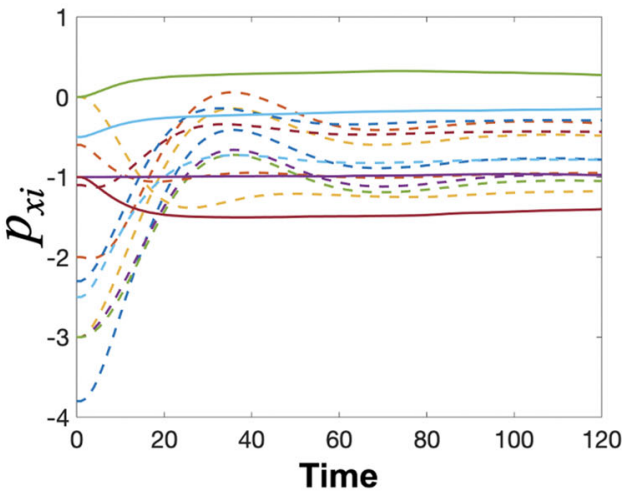

a

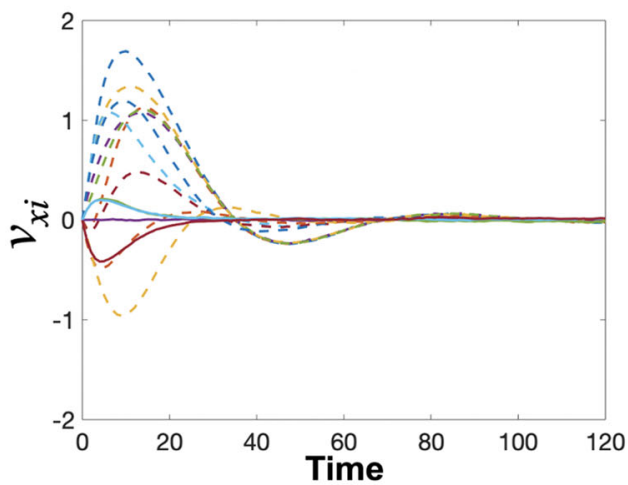

C

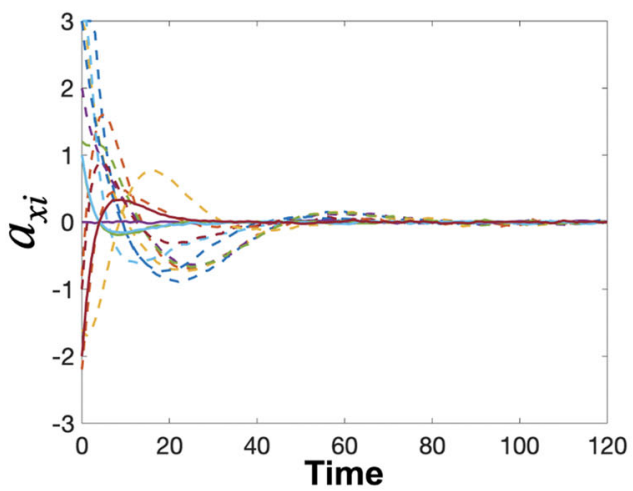

e

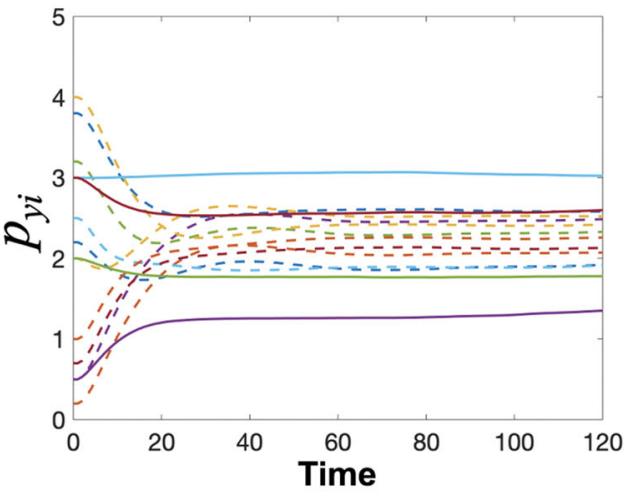

b

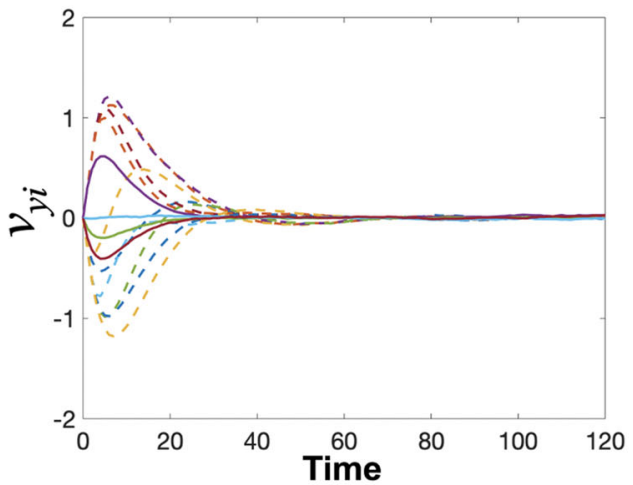

d

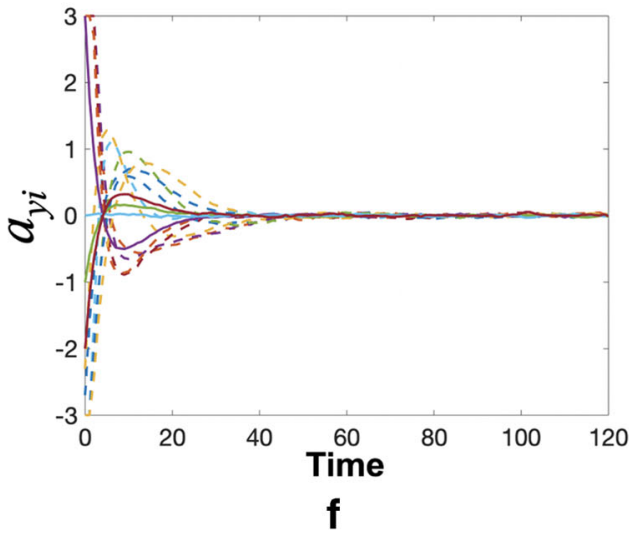

interaction between leaders and followers. For each case, we repeated the experiments 50 times and the initial position of the robots were randomly selected for each run.

A. Number of followers $(N)$ : Assuming all leaders can transmit information to two followers and every follower can receive information from at least one leader. The only difference is the number of followers. We can indicate from Fig. 5a that the value of $T$ increased as an increase in $N$. Also, as shown in Fig. 5b, the value of $d$ was also raised as an increase in $N$, however, with a different trend.
B. Leaders can transmit information to how many followers $\left(N_{l}\right)$ : Assuming there are four followers and every follower can receive information from at least one leader. The only difference is that leaders can transmit information to how many followers. We can see from Fig. 6a that the value of $T$ was decreased as an increase in $N_{l}$. However, the change in the value of $d$ had no strong relationship with the various $N_{l}$.

C. How many followers can receive information from leaders $\left(N_{f}\right)$ : Assuming there are four followers and all leaders can transmit information to one follower. 

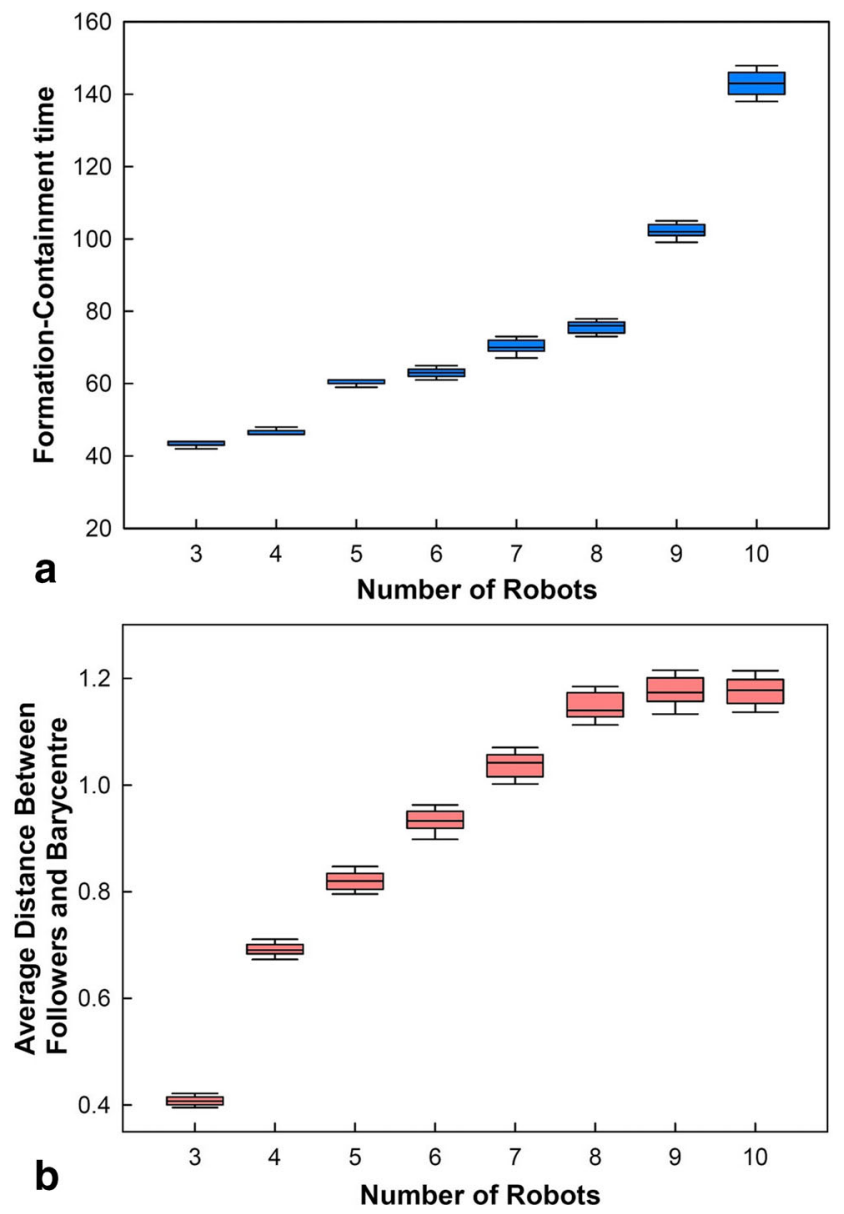

Fig. 5 a Time of formation-containment achievement, $T$, and $\mathbf{b}$ average distance between followers and barycentre, $d$, for number of followers, $N \in\{3,4,5,6,7,8,9,10\}$ robots

The only difference is that the number of followers can receive information from the leaders. We can observe from Fig. $6 \mathrm{~b}$ that the value of $T$ decreased as $N_{f}$ increased. However, the value of $d$ increased as $N_{f}$ increased.

Therefore, we can see that the time for formationcontainment can be affected by both the numbers of followers and the interactions between leaders and followers. The convergence speed slows down when the number of robots increases or the interaction becomes sparse. Hence, the required time for formation-containment will be prolonged. However, the average distance between followers and barycentre is affected mostly by the interaction, which increases as the interaction become sparse. In fact, the trend of the red line in Fig. 6a is mainly due to the interaction since the interaction will become sparse in ring topology as the number of robots increases. If we change to another topology, the performance will be different.
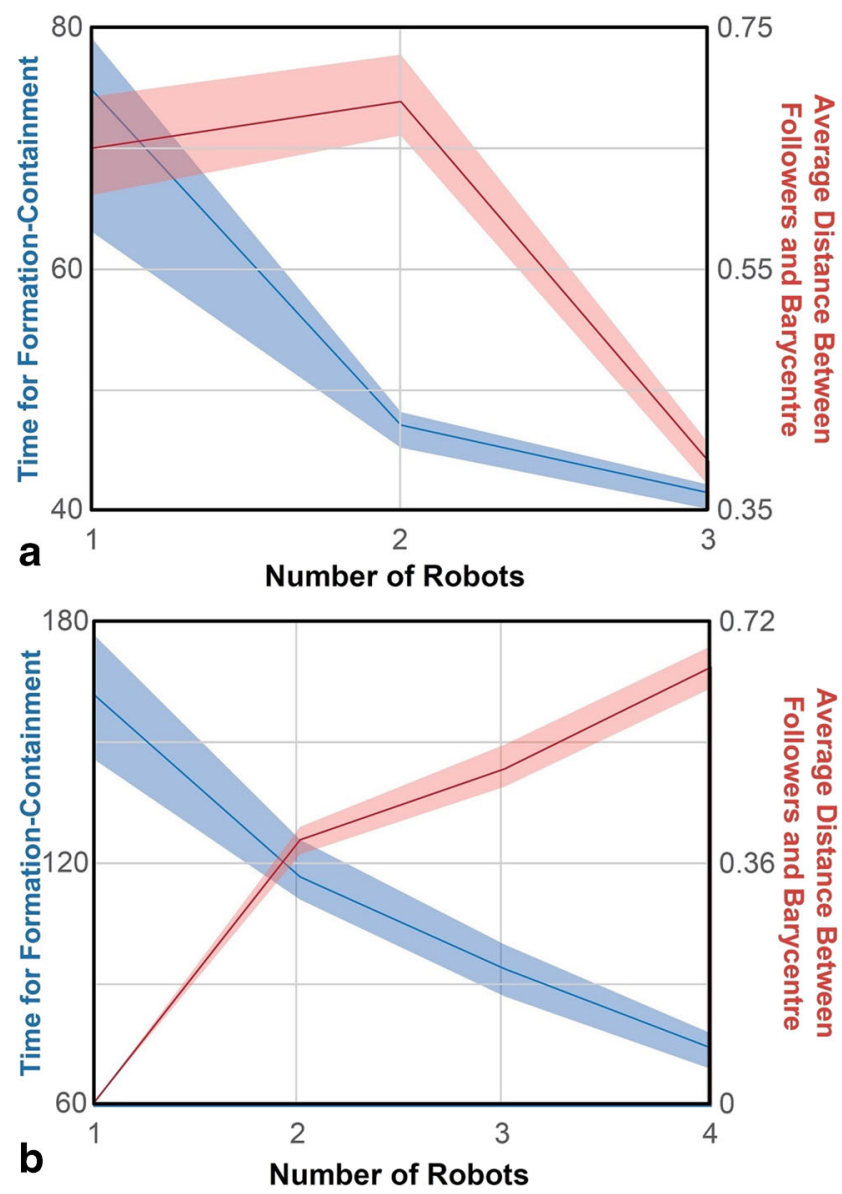

Fig. 6 Time of formation-containment achievement, $T$, and average distance between followers and barycentre, $d$, for a the leaders link to $N_{l} \in\{1,2,3\}$ followers and $\mathbf{b}$ followers number $\left(N_{f} \in\{1,2,3,4\}\right)$ which directly linked to the leaders. The average values of $T$ and $d$ from 50 simulations are indicated by the blue and red colour lines, respectively. The shaded area indicates all the obtained results (between minimum and maximum)

\subsection{Real-Robot Experiments}

\subsubsection{Experimental Setup}

To investigate the performance of the proposed formation scenario, we used a collection of real robots, namely Mona robots which are an open-source swarm robotic platform [45]. Figure 7a shows a Mona robot and its various modules. The robot is based on Arduino AVR architecture with ATMEGA-328 micro-controller. It is actuated with two wheels (with $3.2 \mathrm{~mm}$ diameter), which are differentially driven using two gear-head micro DC motors. The main controller uses PWM (pulse-width modulation) to control the rotational speed of the left and right motors independently. We developed an arena with an overhead camera as shown in Fig. 7b. In the experimental setup, we used a low-cost Microsoft LifeCam Studio Webcam as the swarm localization platform. The position of each 

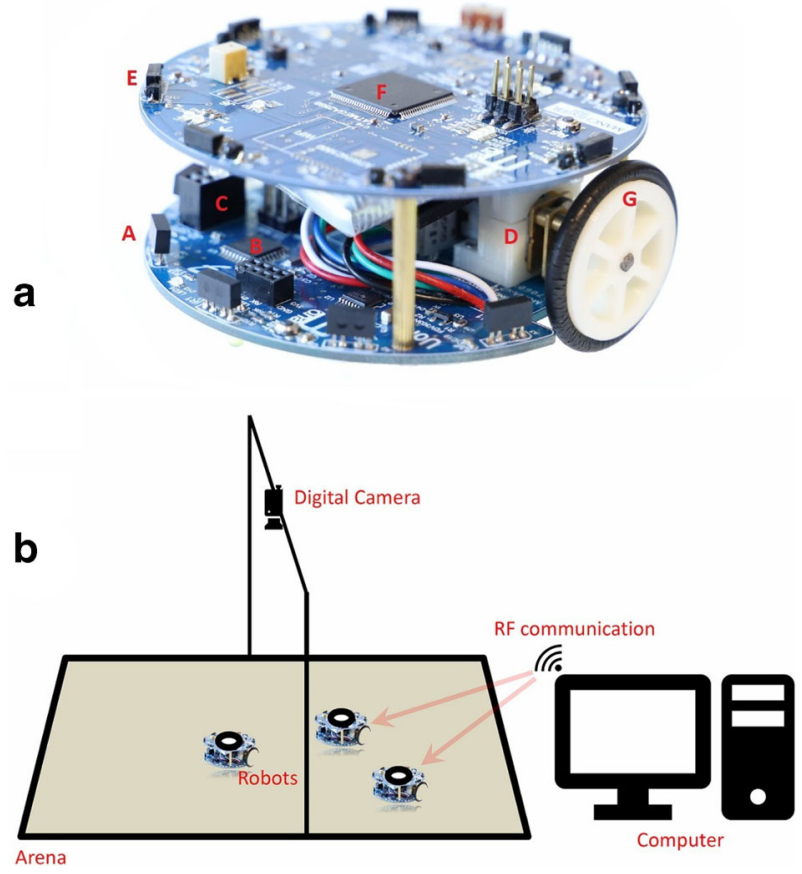

Fig. 7 a Mona Robot, an open-source swarm robotic platform. A) infrared proximity sensors, B) main AVR processor, C) SPI port for RF transceiver. D) gear-head DC micro-motor, E) local IR communication transmitters and encoders, F) communication modules processor, $\mathrm{G}$ ) $32 \mathrm{~mm}$ wheels. b Arena configuration including a PC which tracks the position of robots using digital camera and sends motion commands to the robots using RF communication

robot was continuously tracked by an open-source tracking software developed in [46] with a sampling time of $0.1 \mathrm{~s}$. A time delay of $0.05 \mathrm{~s}$ and a tracking error of $\pm 0.005 \mathrm{~m}$ can be observed during the experiments due to the processing speed of the host computer and the quality of the camera. Hence, the robustness of the swarm system subjected to certain communication delays, actuator noises and inaccuracies of the camera tracking system that can be verified via the experiments.

The control algorithm generates the next position for each robot and transmits this information to them using an RF (radio frequency) module, which is connected to the robots using a serial port. The generated position command contains two bytes for the rotational speeds of the left and right motors. The main controller (micro-controller) of the robots receives this command and translates it to two PWM signals associated with the left and right motors. Details on motion control and kinematics of the robot were presented in [45]. All the mobile robots used in the experiments have the same physical and hardware configuration. The dynamic model in terms of the global coordinates can be described as follows

$$
\begin{aligned}
\dot{p}_{x i} & =v_{i} \cos \theta_{i}, \\
\dot{p}_{y i} & =v_{i} \sin \theta_{i}, \\
\dot{\theta}_{i} & =\omega_{i},
\end{aligned}
$$

where $\left(p_{x i}, p_{y i}\right)$ denotes the position of the $i^{t h}$ robot and $\theta_{i}$ is the orientation. $v_{i}$ and $\omega_{i}$ represent the linear and angular velocities of the $i$ th robot respectively. In order to deal with the nonlinear dynamics appeared in the robot model, the feedback linearization technique [47] was used to transfer the dynamics of the robots Eq. 44 to singleintegrator systems, such that the algorithm proposed in the previous section can be directly applied on the feedback linearized dynamics.

\subsubsection{Results with Real Mobile Robots}

In the real-robot experiment, six Mona robots (4 leaders and 2 followers) were utilised. The goal in these experiments was to make the robots move and then observe the trajectory they followed to achieve the final position. Analysis of this trajectory allowed the functionality of the proposed formation-containment to be verified.

We utilised six robots, where the four leaders are linked by the red dashed lines as shown in Fig. 8, which illustrates trajectory of the four leaders and two followers. In Fig. 8a, the robots are positioned at randomly selected initial states. From Fig. $8 \mathrm{~b}$ to $\mathrm{c}$, it was observed that the robots started to move toward the desired position. The leaders accomplished their formation control in a square, and the followers moved toward the convex hull spanned by the leaders. In Fig. 8d, the formation-containment task is seen to be complete. We can see from Fig. 8 that the leaders formed a square arrangement, that was set as the desired formation, gradually. At the same time, the followers entered the convex hull formed by the leaders, demonstrating that formation-containment was accomplished. Hence, it is clear from these figures that the robots were able to achieve the formation-containment by the control protocol proposed in this study.

In this set of experiment, because time-delays and tracking errors existed in the camera tracking systems, the trace of the robots was not as smooth as might be expected (especially the yellow trace). One of the reasons was that the robots were driven by two motors, there also existed some tracking error between the desired speed and real speed. Furthermore, since the moment they receive the command via RF (radio frequency) module may be delayed because of the wireless connection, and there also exists disturbances (such as friction and the internal interrupts of the robots), further deviation of the tracking was observed. On the another hand, due to the fact that the robots could not be treated as particles (points) in the real-world environment, we activated the collision avoidance function of the robots using artificial potential field methods. The robots changed their route when they discovered their distance between other robots was smaller than the threshold. Despite all of this, the robustness of the control system was shown to 


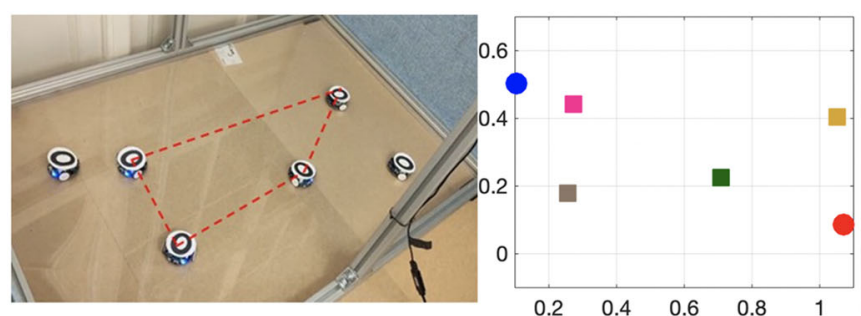

a

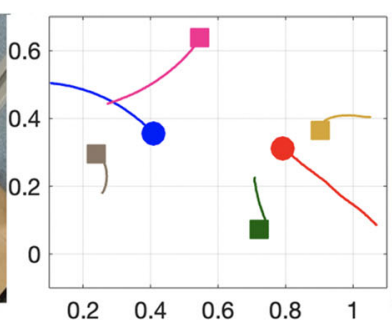

C

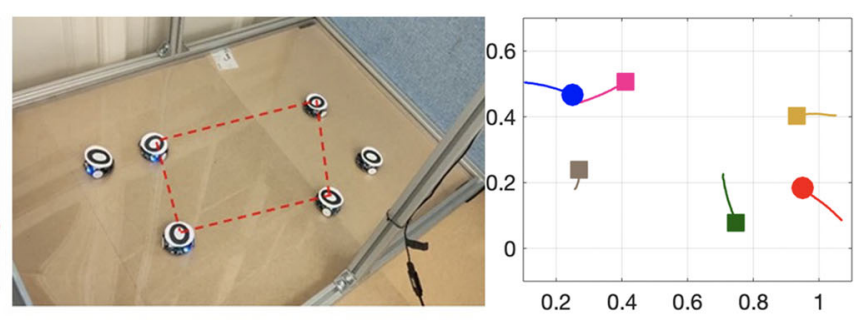

b

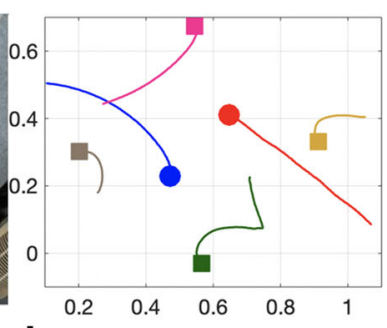

Fig. 8 Selected snapshots of the experiments and the trace with six robots at $\mathbf{a} t=0 \mathrm{~s}, \mathbf{b} t=10 \mathrm{~s}, \mathbf{c} t=20 \mathrm{~s}$ and $\mathbf{d} t=40 \mathrm{~s}$. The leaders are linked with red dashed lines

overcome these difficulties. Hence, the task was completed, and the effectiveness of the proposed controller validated.

According to the results, the control protocol was successfully applied to real robots allowing them to achieve formation-containment. The proposed technique has significant potential to perform more complex behaviors to assist humans in dealing with challenging tasks in the real-world scenarios.

\subsection{Comparisons}

In contrast to the formation tracking problems solved in [25] where there exists only one leader in the swarm system, in this work, we deal with the case when there exist multiple leaders, which bring more challenges to the control system design. Similar containment control problems are discussed in [31, 43], however, for each robot, it requires not only the relative state information from its neighbors, but also the relative formation reference signal which cannot be measured by the distance sensors directly, such that the control scheme is not fully distributed and scalable. On the contrary, the proposed control protocol only requires the relative position measurements, which provides more feasibility in real-world implementation.

In order to show the superior coordination performance under the proposed algorithm, a comparison between the proposed formation-containment protocol and the adaptive controller recently developed in [36] is made. For both controllers, we adopted the same dynamics and conditions as shown in Section 4.3. The initial state of each robot was selected randomly. We repeated experiments 50 times for each controller, where we used $\left\|p-p^{*}\right\|$ ( $p$ is the position vector of every robot, and $p^{*}$ is the final state vector of every robot) to define the tracking error. The performance analysis is illustrated in Fig. 9, where in (a), the yellow area represents the observed results from 50 times experiments of our controller and the solid yellow line is the mean value. In Fig. 9b, the blue area represents results from 50
Fig. 9 Controller performances of $\mathbf{a}$ the proposed protocol in this work and $\mathbf{b}$ the protocol proposed in [36]. The shaded area represents the observed results from 50 times experiments and lines represent the mean value

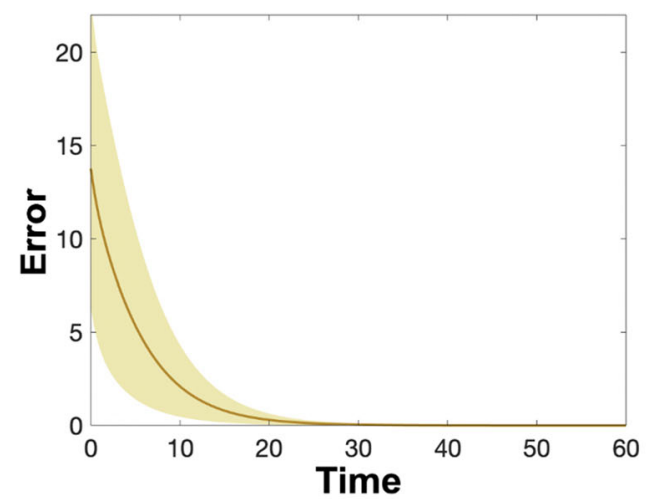

a

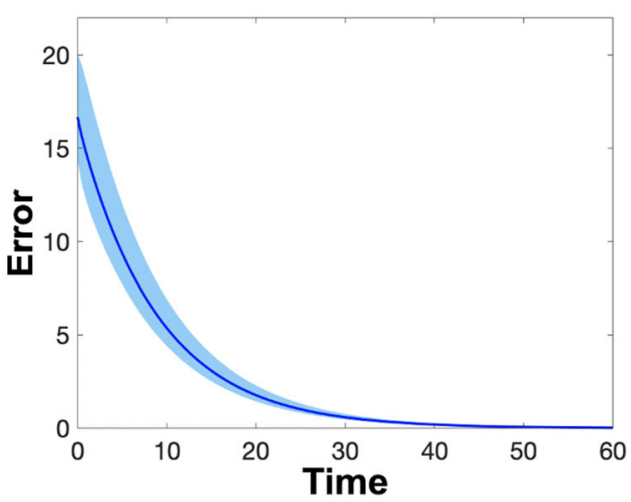

b 
times experiments of the controller proposed in [36] and the solid blue line is the mean value. It can be seen that the convergence time of the proposed protocol in this work is shorter than [36]. Such advantages reveal that our protocol is more efficient in completing formation-containment tasks.

\section{Conclusions}

In conclusion, a novel formation-containment control protocol design was proposed that makes the leaders converge to the desired formation, and the followers move to the convex hull spanned by the leaders. Simulations were performed to verify the control design algorithm and analyzed some of the factors which affected the formationcontainment performance. Finally, we applied our theorem to a real-world scenario by performing an experiment using multiple mobile robots. It can be seen that the proposed coordination framework can be used in precision agriculture applications where the leaders act as markers, indicating an area of interest and the followers are robots that interact with the plants. In the future, nonlinear dynamics of the robot and time-delay of the sensors will be taken into consideration when designing the distributed protocol. Furthermore, robust control techniques [48] will also be exploited to guarantee reliable performance.

Author Contributions KW completed the main theory and wrote the draft. JH proposed the research idea and conducted the experiments. BL and FA provided hardware equipment, improved the manuscript and supervised the overall project. All the authors analyzed the theory, discussed the results, reviewed and approved the paper.

Funding This work was supported by EU H2020-FET-OPEN Robocoenosis project [grant number 899520] and the UK Engineering and Physical Sciences Research Council (EPSRC) projects RAIN (EP/R026084/1) and RNE (EP/P01366X/1).

Availability of data and materials All research data supporting this study are included in the manuscript.

\section{Declarations}

Ethical Approval The authors state that this research complies with ethical standards.

Conflict of Interests The authors declare that there is no conflict of interest.

Open Access This article is licensed under a Creative Commons Attribution 4.0 International License, which permits use, sharing, adaptation, distribution and reproduction in any medium or format, as long as you give appropriate credit to the original author(s) and the source, provide a link to the Creative Commons licence, and indicate if changes were made. The images or other third party material in this article are included in the article's Creative Commons licence, unless indicated otherwise in a credit line to the material. If material is not included in the article's Creative Commons licence and your intended use is not permitted by statutory regulation or exceeds the permitted use, you will need to obtain permission directly from the copyright holder. To view a copy of this licence, visit http://creativecommons. org/licenses/by/4.0/.

\section{References}

1. Schranz, M., Di Caro, G.A., Schmickl, T., Elmenreich, W., Arvin, F., Şekercioğlu, A., Sende, M.: Swarm intelligence and cyberphysical systems: concepts, challenges and future trends. Swarm Evol. Comput. 60, 100762 (2020)

2. Ferrante, E., Turgut, A.E., Dorigo, M., Huepe, C.: Elasticity-based mechanism for the collective motion of self-propelled particles with springlike interactions: a model system for natural and artificial swarms. Phys. Rev. Lett. 111(26), 268302 (2013)

3. Jang, I., Hu, J., Arvin, F., Carrasco, J., Lennox, B.: Omnipotent virtual giant for remote human-swarm interaction. arXiv:1903.10064 (2019)

4. Ordaz-Rivas, E., Rodriguez-Liñan, A., Aguilera-Ruíz, M., TorresTreviño, L.: Collective tasks for a flock of robots using influence factor. J. Intell. Robot. Syst. 94(2), 439-453 (2019)

5. Turgut, A.E., Çelikkanat, H., Gökçe, F., Şahin, E.: Self-organized flocking in mobile robot swarms. Swarm Intell. 2(2-4), 97-120 (2008)

6. Arvin, F., Turgut, A.E., Krajník, T., Yue, S.: Investigation of cuebased aggregation in static and dynamic environments with a mobile robot swarm. Adapt. Behav. 24(2), 102-118 (2016)

7. Schmickl, T., Hamann, H.: Beeclust: a swarm algorithm derived from honeybees. Bio-inspired computing and communication networks, 95-137 (2011)

8. Guo, H., Meng, Y.: Distributed reinforcement learning for coordinate multi-robot foraging. J. Intell. Robot. Syst. 60(3-4), 531-551 (2010)

9. Pini, G., Brutschy, A., Pinciroli, C., Dorigo, M., Birattari, M.: Autonomous task partitioning in robot foraging: an approach based on cost estimation. Adapt. Behav. 21(2), 118-136 (2013)

10. Daltorio, K.A., Tietz, B.R., Bender, J.A., Webster, V.A., Szczecinski, N.S., Branicky, M.S., Ritzmann, R.E., Quinn, R.D.: A model of exploration and goal-searching in the cockroach, blaberus discoidalis. Adapt. Behav. 21(5), 404-420 (2013)

11. Groß, R., Dorigo, M.: Evolution of solitary and group transport behaviors for autonomous robots capable of self-assembling. Adapt. Behav. 16(5), 285-305 (2008)

12. Bai, H., Wen, J.T.: Cooperative load transport: a formation-control perspective. IEEE Trans. Robot. 26(4), 742-750 (2010)

13. Chen, J., Gauci, M., Li, W., Kolling, A., Groß, R.: Occlusionbased cooperative transport with a swarm of miniature mobile robots. IEEE Trans. Robot. 31(2), 307-321 (2015)

14. Hu, J., Turgut, A.E., Krajník, T., Lennox, B., Arvin, F.: Occlusionbased coordination protocol design for autonomous robotic shepherding tasks. IEEE Trans. Cogn. Dev. Syst. (2020)

15. Rubenstein, M., Cornejo, A., Nagpal, R.: Programmable selfassembly in a thousand-robot swarm. Science 345(6198), 795-799 (2014)

16. Hu, J., Niu, H., Carrasco, J., Lennox, B., Arvin, F.: Voronoi-based multi-robot autonomous exploration in unknown environments via deep reinforcement learning. IEEE Trans. Veh. Technol. 69(12), 14413-14423 (2020)

17. McGuire, K., De Wagter, C., Tuyls, K., Kappen, H., de Croon, G.: Minimal navigation solution for a swarm of tiny flying robots to explore an unknown environment. Sci. Robot. 4(35), eaaw9710 (2019) 
18. Albani, D., IJsselmuiden, J., Haken, R., Trianni, V.: Monitoring and mapping with robot swarms for agricultural applications. In: 4th IEEE International Conference on Advanced Video and Signal Based Surveillance (AVSS), pp. 1-6 (2017)

19. Dorigo, M., Theraulaz, G., Trianni, V.: Reflections on the future of swarm robotics. Sci. Robot. 5, eabe4385 (2020)

20. Capitan, J., Merino, L., Ollero, A.: Cooperative decision-making under uncertainties for multi-target surveillance with multiples uavs. J. Intell. Robot. Syst. 84(1-4), 371-386 (2016)

21. Hu, J., Bhowmick, P., Lanzon, A.: Distributed adaptive timevarying group formation tracking for multiagent systems with multiple leaders on directed graphs. IEEE Trans. Control Netw. Syst. 7(1), 140-150 (2020)

22. Harder, S.A., Lauderbaugh, L.K.: Formation specification for control of active agents using artificial potential fields. J. Intell. Robot. Syst. 95(2), 279-290 (2019)

23. Rojo-Rodriguez, E.G., Garcia, O., Ollervides, E., ZambranoRobledo, P., Espinoza-Quesada, E.: Robust consensus-based formation flight for multiple quadrotors. J. Intell. Robot. Syst. 93(1-2), 213-226 (2019)

24. Mei, F., Wang, H., Yao, Y., Fu, J., Yuan, X., Yu, W.: Robust second-order finite-time formation control of heterogeneous multi-agent systems on directed communication graphs. IET Control Theory Appl. 14(6), 816-823 (2020)

25. Hong, Y., Chen, G., Bushnell, L.: Distributed observers design for leader-following control of multi-agent networks. Automatica 44(3), 846-850 (2008)

26. Dimarogonas, D.V., Johansson, K.H.: Further results on the stability of distance-based multi-robot formations. In: 2009 American Control Conference, pp. 2972-2977. IEEE (2009)

27. Hu, J., Bhowmick, P., Arvin, F., Lanzon, A., Lennox, B.: Cooperative control of heterogeneous connected vehicle platoons: an adaptive leader-following approach. IEEE Robot. Autom. Lett. 5(2), 977-984 (2020)

28. Chen, J., Shi, Z., Zhong, Y.: Robust formation tracking for uncertain multi-agent systems with unknown leader input. IET Control Theory Appl. 14(4), 646-653 (2020)

29. Ji, M., Ferrari-Trecate, G., Egerstedt, M., Buffa, A.: Containment control in mobile networks. IEEE Trans. Autom. Control 53(8), 1972-1975 (2008)

30. Cao, Y., Stuart, D., Ren, W., Meng, Z.: Distributed containment control for multiple autonomous vehicles with double-integrator dynamics: algorithms and experiments. IEEE Trans. Control Syst. Technol. 19(4), 929-938 (2010)

31. Dong, X., Shi, Z., Lu, G., Zhong, Y.: Output containment analysis and design for high-order linear time-invariant swarm systems. Int. J. Robust Nonlinear Control 25(6), 900-913 (2015)

32. Li, Z., Ren, W., Liu, X., Fu, M.: Distributed containment control of multi-agent systems with general linear dynamics in the presence of multiple leaders. Int. J. Robust Nonlinear Control 23(5), 534547 (2013)

33. Ferrari-Trecate, G., Egerstedt, M., Buffa, A., Ji, M.: Laplacian sheep: a hybrid, stop-go policy for leader-based containment control. In: International Workshop on Hybrid Systems: Computation and Control, pp. 212-226. Springer (2006)

34. Liu, H., Cheng, L., Tan, M., Hou, Z., Cao, Z., Wang, M.: Containment control with multiple interacting leaders under switching topologies. In: Proceedings of the 32nd Chinese Control Conference, pp. 7093-7098. IEEE (2013)

35. Dong, X., Shi, Z., Lu, G., Zhong, Y.: Formation-containment analysis and design for high-order linear time-invariant swarm systems. Int. J. Robust Nonlinear Control 25(17), 3439-3456 (2015)
36. Hu, J., Bhowmick, P., Lanzon, A.: Two-layer distributed formation-containment control strategy for linear swarm systems: algorithm and experiments. Int. J. Robust Nonlinear Control 30(16), 6433-6453 (2020)

37. Liu, Z., West, C., Lennox, B., Arvin, F.: Local bearing estimation for a swarm of low-cost miniature robots. Sensors 20(11), 3308 (2020)

38. Godsil, C., Royle, G.F.: Algebraic Graph Theory, vol. 207. Springer Science \& Business Media, Berlin (2013)

39. Fathian, K., Rachinskii, D.I., Summers, T.H., Spong, M.W., Gans, N.R.: Distributed formation control under arbitrarily changing topology. In: 2017 American Control Conference (ACC), pp. 271278. IEEE (2017)

40. Meng, Z., Ren, W., You, Z.: Distributed finite-time attitude containment control for multiple rigid bodies. Automatica 46(12), 2092-2099 (2010)

41. Fathian, K., Safaoui, S., Summers, T.H., Gans, N.R.: Robust distributed planar formation control for higher-order holonomic and nonholonomic agents. arXiv:1807.11058 (2018)

42. Liberzon, D.: Switching in Systems and Control. Springer Science \& Business Media, Berlin (2003)

43. Dong, X., Xi, J., Lu, G., Zhong, Y.: Containment analysis and design for high-order linear time-invariant singular swarm systems with time delays. Int. J. Robust Nonlinear Control 24(7), 11891204 (2014)

44. Grieve, B.D., Duckett, T., Collison, M., Boyd, L., West, J., Yin, H., Arvin, F., Pearson, S.: The challenges posed by global broadacre crops in delivering smart agri-robotic solutions: a fundamental rethink is required. Glob. Food Secur. 23, 116-124 (2019)

45. Arvin, F., Espinosa, J., Bird, B., West, A., Watson, S., Lennox, B.: Mona: an affordable open-source mobile robot for education and research. J. Intell. Robot. Syst. 94(3-4), 761-775 (2019)

46. Krajník, T., Nitsche, M., Faigl, J., Vaněk, P., Saska, M., Přeučil, L., Duckett, T., Mejail, M.: A practical multirobot localization system. J. Intell. Robot. Syst. 76(3-4), 539-562 (2014)

47. Tzafestas, S.G.: Introduction to Mobile Robot Control. Elsevier (2013)

48. Hu, J., Bhowmick, P.: A consensus-based robust secondary voltage and frequency control scheme for islanded microgrids. Int. J. Electr. Power Energy Syst. 116, 105575 (2020)

Publisher's Note Springer Nature remains neutral with regard to jurisdictional claims in published maps and institutional affiliations.

Kefan Wu received B.Sc degree from Lanzhou University in 2016 and M.Sc degree from Wuhan University in 2019. He is currently working toward the Ph.D degree in Electrical and Electronic Engineering at the University of Manchester. His research interests include swarm robotics and control theory.

Junyan Hu received B.Eng degree in Automation from Hefei University of Technology in 2015 and Ph.D degree in Electrical and Electronic Engineering from the University of Manchester in 2020. Dr. $\mathrm{Hu}$ is currently a Postdoctoral Research Associate in Robotics at the University of Manchester. His research interests include multi-robot coordination, cooperative control, autonomous systems and swarm intelligence. 
Barry Lennox is a Professor of Applied Control and Nuclear Engineering Decommissioning and holds a Royal Academy of Engineering Chair in Emerging Technologies. He is Director of the Robotics and Artificial Intelligence for Nuclear (RAIN) Robotics Hub and Research Director of the Dalton Cumbrian Facility. He is a Fellow of the Royal Academy of Engineering, Senior Member of the IEEE, Fellow of the IET and InstMC, and a Chartered Engineer. $\mathrm{He}$ is an expert in applied control and its use in robotics and process operations and has considerable experience in transferring leading edge technology into industry.
Farshad Arvin is an Assistant Professor in Robotics at the University of Manchester, UK since 2018. He received his BSc degree in Computer Engineering in 2004, MSc degree in Computer Systems Engineering in 2010, and $\mathrm{PhD}$ in Computer Science in 2015. Farshad visited several leading institutes including Artificial Life Laboratory in University of Graz, Institute of Microelectronics at Tsinghua University in Beijing, and Italian Institute of Technology (iit) in Genoa as a Senior Visiting Research Scholar. His research interests include Swarm Robotics and Autonomous Multi-agent Systems. He is the founding director of the Swarm \& Computation Intelligence Laboratory (SwaCIL) formed in 2018. 